\title{
A sequential approach to the convolution of Roumieu ultradistributions
}

\section{Svetlana Mincheva-Kamińska ${ }^{1}$}

Received: 30 March 2020 / Accepted: 1 July 2020/Published online: 22 December 2020

(C) The Author(s) 2020

\section{Abstract}

We consider several general sequential conditions for convolvability of two Roumieu ultradistributions on $\mathbb{R}^{d}$ in the space $\mathcal{D}^{\prime\left\{M_{p}\right\}}$ and prove that they are equivalent to the convolvability of these ultradistributions in the sense of Pilipovic and Prangoski. The discussed conditions, based on two classes $\mathbb{U}^{\left\{M_{p}\right\}}$ and $\overline{\mathbb{U}}^{\left\{M_{p}\right\}}$ of approximate units and corresponding sequential conditions of integrability of Roumieu ultradistributions, are analogous to the known convolvability conditions in the space $\mathcal{D}^{\prime}$ of distributions and in the space $\mathcal{D}^{\prime\left(M_{p}\right)}$ of ultradistributions of Beurling type. Moreover, the following property of the convolution and ultradifferential operator $P(D)$ of class $\left\{M_{p}\right\}$ is proved: if $S, T \in \mathcal{D}^{\prime\left\{M_{p}\right\}}\left(\mathbb{R}^{d}\right)$ are convolvable, then

$$
P(D)(S * T)=(P(D) S) * T=S *(P(D) T) .
$$

Keywords Ultradistributions - Convolution of ultradistributions - Approximate unit · Ultradifferential operator

Mathematics Subject Classification 46F05 - 46F10 - 46E10

Communicated by Matjaz Omladic.

Svetlana Mincheva-Kamińska

minczewa@ur.edu.pl

1 Faculty of Mathematics and Natural Sciences, Institute of Mathematics, University of Rzeszów, Prof. Pigonia 1, 35-310 Rzeszów, Poland 


\section{Introduction}

Deep investigations of the convolution of two ultradistributions of Roumieu type (that we call shorter Roumieu ultradistributions) in the non-quasianalytic case were carried out via $\varepsilon$-tensor product by Pilipović and Prangoski in [19] and, with important improvements, by Dimovski et al. in [7]. The authors gave there general functional definitions and proved fundamental results on convolvability and the convolution of Roumieu ultradistributions in a way analogous to the known general approaches of Chevalley and Schwartz in case of distributions. For other aspects of the theory, see, e.g., [1, 2, 4, 6, 8, 20, 24]. See also the recent article [21] for results concerning the quasianalytic case.

The aim of this paper is to discuss sequential conditions playing a similar role in the study of the convolution of Roumieu ultradistributions to those used in the sequential theories of the convolution of distributions (see [5, 9, 15, 23]) and ultradistributions of Beurling type (see [1, 10,11]). The conditions are based on two types of $\mathfrak{R}$-approximate units (Definitions 4.2 and 4.3), being the counterparts of the approximate units in the sense of Dierolf and Voigt (see [5]). The respective classes $\mathbb{U}^{\left\{M_{p}\right\}}$ and $\overline{\mathbb{U}}^{\left\{M_{p}\right\}}$ of $\mathfrak{R}$-approximate units are used in a sequential characterization of integrable Roumieu ultradistributions (see [16]), analogous to that proved by Pilipović in [18] in case of integrable ultradistributions of Beurling type. As a consequence, we give in this paper several sequential definitions of the convolution of Roumieu ultradistributions (Definition 7.2). We prove in Theorem 7.1, that all our sequential definitions of the convolution of Roumieu ultradistributions are equivalent to those given in [19] and [7].

An important application of the notion of $\mathfrak{R}$-approximate units is presented in the proof of Theorem 8.1, describing a non-trivial property of the convolution of Roumieu ultradistributions and ultradifferential operators of the class $\left\{M_{p}\right\}$.

It is worth to recall that Pilipović in [18] used a different class of approximate units to prove the same property in case of the convolution of ultradistributions of Beurling type. Our proof of Theorem 8.1 is based on similar ideas but discussions concerning the class $\mathfrak{R}$ play an essential role in our case.

\section{Preliminaries}

We consider complex-valued $\mathcal{C}^{\infty}$-functions and Roumieu ultradistributions defined on $\mathbb{R}^{d}$ (or on an open subset of $\mathbb{R}^{d}$ ) using the standard multi-dimensional notation in $\mathbb{R}^{d}$.

To mark the dimension of $\mathbb{R}^{d}$, which is essential in some situations, we denote the considered spaces of test functions and the corresponding spaces of Roumieu ultradistributions simply by adding the index $d$ at the end of the respective symbol. Moreover, if necessary, the constant function 1 on $\mathbb{R}^{d}$ will be denoted by $1_{d}$ and the value of $T \in \mathcal{D}_{d}^{\prime\left\{M_{p}\right\}}$ on $\varphi \in \mathcal{D}_{d}^{\left\{M_{p}\right\}}$ by $\langle T, \varphi\rangle_{d}$. 
The spaces of test functions and Roumieu ultradistributions are defined by a given sequence $\left(M_{p}\right)_{p \in \mathbb{N}_{0}}$ of positive numbers. Usually some of the following conditions are imposed on the sequence $\left(M_{p}\right)$ :

(M.1) $\quad M_{p}^{2} \leq M_{p-1} M_{p+1}, \quad p \in \mathbb{N}$;

(M.2) $\quad M_{p} \leq A H^{p} M_{q} M_{p-q}, \quad p, q \in \mathbb{N}_{0}, q \leq p$;

(M.2' $\quad M_{p} \leq A H^{p} M_{p-1}, \quad p \in \mathbb{N}$;

(M.3) $\quad \sum_{p=q+1}^{\infty} M_{p-1} M_{p}^{-1} \leq A q M_{q} M_{q+1}^{-1}, \quad q \in \mathbb{N}$;

$\left(\mathrm{M} .3^{\prime}\right) \quad \sum_{p=1}^{\infty} M_{p-1} M_{p}^{-1}<\infty$,

for certain constants $A>0$ and $H>0$. We can and will assume that $H \geq 1$.

Clearly, conditions (M.2') and (M.3') are particular cases of conditions (M.2) and (M.3), respectively.

For simplicity, we will assume in the whole paper that the sequence $\left(M_{p}\right)$ satisfies the three conditions (M.1), (M.2) and (M.3), not discussing which of them can be weakened or omitted in the formulations of presented theorems.

It follows, by induction, from (M.1) that $M_{p} \cdot M_{q} \leq M_{0} M_{p+q}$ for $p, q \in \mathbb{N}_{0}$ (see [16]). Under the assumption that $M_{0}=1$, which we adopt hereinafter for simplicity, the last inequality admits the form:

$$
M_{p} \cdot M_{q} \leq M_{p+q}, \quad p, q \in \mathbb{N}_{0} .
$$

It will be convenient to extend the sequence $\left(M_{p}\right)_{p \in \mathbb{N}_{0}}$ to $\left(M_{k}\right)_{k \in \mathbb{N}_{0}^{d}}$ by means of the formula:

$$
M_{k}:=M_{k_{1}+\ldots+k_{d}}, \quad k=\left(k_{1}, \ldots, k_{d}\right) \in \mathbb{N}_{0}^{d} .
$$

Due to the extended notation, we immediately get the extended version of inequality (2.1):

$$
M_{j} \cdot M_{k} \leq M_{j+k}, \quad j, k \in \mathbb{N}_{0}^{d} .
$$

The associated function of the sequence $\left(M_{p}\right)$ is given by

$$
M(\rho)=\sup _{p \in \mathbb{N}_{0}} \log _{+} \frac{\rho^{p}}{M_{p}}, \quad \rho>0 .
$$

For an arbitrary $k=\left(k_{1}, \ldots, k_{d}\right) \in \mathbb{N}_{0}^{d}$ denote by $D^{k}$ the differential operator of the form

$$
D^{k}=D_{1}^{k_{1}} \cdots D_{d}^{k_{d}}:=\left(\frac{1}{i} \frac{\partial}{\partial x_{1}}\right)^{k_{1}} \cdots\left(\frac{1}{i} \frac{\partial}{\partial x_{d}}\right)^{k_{d}} .
$$

An essential role in our considerations will be played by Komatsu's lemma proved in [14] (see Lemma 3.4 and Proposition 3.5) in which numerical sequences monotonously increasing to infinity are involved. The class of such sequences $\left(r_{p}\right)_{p \in \mathbb{N}_{0}}$ (with $r_{0}=1$ ) was denoted by $\mathfrak{R}$ in [19] and [7] and we preserve this notation in our paper. 
For every $\left(r_{p}\right) \in \mathfrak{R}$ we call $\left(R_{p}\right)$ the product sequence corresponding to $\left(r_{p}\right)$ if its elements are of the form $R_{p}:=\prod_{i=0}^{p} r_{i}$ for $p \in \mathbb{N}_{0}$ (i.e., $R_{0}=1$ ).

Let us recall Komatsu's lemma in the following equivalent form which emphasizes the symmetry of two assertions:

Lemma 2.1 Let $\left(a_{k}\right)_{k \in \mathbb{N}_{0}}$ be a sequence of nonnegative numbers.

(I) The following two conditions are equivalent:

$$
\begin{aligned}
& \left(A_{1}\right) \quad \exists_{h>0} \quad \sup _{k \in \mathbb{N}_{0}} \frac{a_{k}}{h^{k}}<\infty, \\
& \left(B_{1}\right) \quad \forall_{\left(r_{k}\right) \in \mathfrak{R}} \sup _{k \in \mathbb{N}_{0}} \frac{a_{k}}{R_{k}}<\infty ;
\end{aligned}
$$

(II) the following two conditions are equivalent:

$$
\begin{aligned}
& \left(A_{2}\right) \quad \forall_{h>0} \quad \sup _{k \in \mathbb{N}_{0}}\left(h^{k} a_{k}\right)<\infty, \\
& \left(B_{2}\right) \quad \exists\left(r_{k}\right) \in \mathfrak{R} \sup _{k \in \mathbb{N}_{0}}\left(R_{k} a_{k}\right)<\infty,
\end{aligned}
$$

where $\left(R_{k}\right)$ is the product sequence corresponding to the sequence $\left(r_{k}\right) \in \mathfrak{R}$.

Remark 2.1 The above lemma can be easily extended to the $d$-dimensional version concerning sequences $\left(a_{k}\right)_{k \in \mathbb{N}_{0}^{d}}$ of nonnegative numbers.

It is worth noticing that Lemma 2.1 delivers two simple characterizations (dual to each other): $1^{\circ}$ of slowly increasing sequences (i.e., satisfying $\left(A_{1}\right)$ ), $2^{\circ}$ of rapidly decreasing sequences (i.e., satisfying $\left(A_{2}\right)$ ) of nonnegative numbers. They are expressed through respective properties of sequences, described by product sequences corresponding to sequences of the class $\mathfrak{R}$.

In what follows we will also apply the following simple lemma (see [16]):

Lemma 2.2 For every $\left(r_{p}\right) \in \mathfrak{R}$, the following inequality holds:

$$
R_{|k|} \cdot R_{|l|} \leq R_{|k+l|}, \quad k, l \in \mathbb{N}_{0}^{d},
$$

where $\left(R_{p}\right)$ is the product sequence corresponding to $\left(r_{p}\right)$.

It will be convenient to use for $\lambda>0$ and $\left(r_{p}\right) \in \mathfrak{R}$ the following notation:

$$
\lambda\left(r_{p}\right)=\left(\bar{r}_{p}\right), \text { where } \bar{r}_{0}=1 \text { and } \bar{r}_{p}=\lambda r_{p} \text { for } p \in \mathbb{N} .
$$

Clearly, if $\left(r_{p}\right) \in \mathfrak{R}$, then $\lambda\left(r_{p}\right) \in \mathfrak{R}$ for $\lambda \geq 1$. Moreover, $\lambda\left(r_{p}\right) \in \mathfrak{R}$ for $0<\lambda<1$ in case $\left(r_{p}\right) \in \mathfrak{R}$ and $r_{1}>\lambda^{-1}$.

\section{Ultradifferentiable functions}

For a given complex-valued function $\varphi$ on $\mathbb{R}^{d}$ and a compact set $K$ in $\mathbb{R}^{d}$ denote 


$$
\|\varphi\|_{\infty}:=\sup _{x \in \mathbb{R}^{d}}|\varphi(x)| ; \quad\|\varphi\|_{K}:=\sup _{x \in K}|\varphi(x)| .
$$

For a given sequence $\left(M_{p}\right)$, a regular compact set $K$ in $\mathbb{R}^{d}$ and $h>0$, the symbol $\mathcal{E}_{K, h, d}^{\left\{M_{p}\right\}}$ will mean the locally convex space (1.c.s.) of all $\mathcal{C}^{\infty}$-functions $\varphi$ on $\mathbb{R}^{d}$ such that

$$
q_{K, h}(\varphi):=\sup _{k \in \mathbb{N}_{0}^{d}} \frac{\left\|D^{k} \varphi\right\|_{K}}{h^{|k|} M_{k}}<\infty,
$$

with the topology defined by the semi-norm $q_{K, h}$ given above, while the symbol $\mathcal{D}_{K, h, d}^{\left\{M_{p}\right\}}$ will mean the Banach space of all $\mathcal{C}^{\infty}$-functions $\varphi$ satisfying (3.1) and having supports contained in $K$, with the topology of the norm $q_{K, h}$ in (3.1).

For a fixed sequence $\left(M_{p}\right)$, we consider the following locally convex spaces of ultradifferentiable functions on $\mathbb{R}^{d}$ :

$$
\begin{aligned}
& \mathcal{D}_{K, d}^{\left\{M_{p}\right\}}:=\lim _{h \rightarrow \infty} \mathcal{D}_{K, h, d}^{\left\{M_{p}\right\}} ; \quad \mathcal{D}_{d}^{\left\{M_{p}\right\}}:=\underset{K \subset \subset \mathbb{R}^{\Perp}}{\lim _{K, d}} \mathcal{D}_{\left.K M_{p}\right\}}^{\longrightarrow} ; \\
& \mathcal{E}_{d}^{\left\{M_{p}\right\}}:=\lim _{K \subset \subset \mathbb{R}^{\Perp}} \underset{h \rightarrow \infty}{\lim _{h \rightarrow h}} \mathcal{E}_{K, h}^{\left\{M_{p}\right\}},
\end{aligned}
$$

where the symbol $K \subset \subset \mathbb{R}^{d}$ means that compact sets $K$ grow up to $\mathbb{R}^{d}$.

Moreover, for a given $\left(M_{p}\right)$, we define

$$
\mathcal{D}_{L^{\infty}, d}^{\left\{M_{p}\right\}}:=\underset{h \rightarrow \infty}{\lim _{h \rightarrow \infty}} \mathcal{D}_{L^{\infty}, h, d}^{\left\{M_{p}\right\}},
$$

where $\mathcal{D}_{L^{\infty}, h, d}^{\left\{M_{p}\right\}}$ is the Banach space of all $\mathcal{C}^{\infty}$-functions $\varphi$ on $\mathbb{R}^{d}$ such that

$$
\|\varphi\|_{\infty, h}:=\sup \left\{\left(h^{k} M_{k}\right)^{-1}\left\|D^{k} \varphi\right\|_{\infty}: k \in \mathbb{N}_{0}^{d}\right\}<\infty
$$

with the norm $\|\cdot\|_{\infty, h}$ defined above.

For a given regular compact set $K \subset \mathbb{R}^{d}$ and given sequences $\left(M_{p}\right)$ and $\left(r_{p}\right) \in \mathfrak{R}$, we denote by $\mathcal{D}_{K,\left(r_{p}\right), d}^{\left\{M_{p}\right\}}$ the Banach space of all $\mathcal{C}^{\infty}$-functions $\varphi$ on $\mathbb{R}^{d}$ having supports contained in $K$ such that

$$
\|\varphi\|_{K,\left(r_{p}\right)}:=\sup _{k \in \mathbb{N}_{0}^{d}} \frac{\left\|D^{k} \varphi\right\|_{K}}{R_{|k|} M_{k}}<\infty
$$

with the norm $\|\cdot\|_{K,\left(r_{p}\right)}$ defined above.

The following result is essentially due to Komatsu [14] (see also [4, 16]), since it is a consequence of his Lemma 2.1 recalled above.

Proposition 3.1 We have the equality 


$$
\mathcal{D}_{K, d}^{\left\{M_{p}\right\}}={\underset{\left(r_{p}\right) \in \mathfrak{R}}{\longleftarrow}}_{\lim _{K,\left(r_{p}\right), d}}^{\left\{M_{p}\right\}},
$$

where the space $\mathcal{D}_{K, d}^{\left\{M_{p}\right\}}$ is defined in (3.2).

For given $\left(M_{p}\right)$ and $\left(r_{p}\right) \in \mathfrak{R}$, we denote by $\mathcal{D}_{L^{\infty},\left(r_{p}\right), d}^{\left\{M_{p}\right\}}$ the Banach space of all $\mathcal{C}^{\infty}$ functions $\varphi$ on $\mathbb{R}^{d}$ such that

$$
\|\varphi\|_{\left(r_{p}\right)}:=\sup _{k \in \mathbb{N}_{0}^{d}} \frac{\left\|D^{k} \varphi\right\|_{\infty}}{R_{|k|} M_{k}}<\infty,
$$

with the norm $\|\cdot\|_{\left(r_{p}\right)}$ defined in (3.6).

For a given sequence $\left(M_{p}\right)$, the following projective description of the space $\mathcal{D}_{L^{\infty}, d}^{\left\{M_{p}\right\}}$ follows from the results proved in $[3,7,21]$ :

$$
\mathcal{D}_{L^{\infty}, d}^{\left\{M_{p}\right\}}={\underset{\left(r_{p}\right) \in \mathfrak{R}}{\longleftarrow}}_{\lim _{L^{\infty},\left(r_{p}\right), d}}^{\left\{M_{p}\right\}}
$$

where the equality holds in the sense of 1.c.s.

We denote by $\dot{\mathcal{B}}_{d}^{\left\{M_{p}\right\}}$ the completion of $\mathcal{D}_{d}^{\left\{M_{p}\right\}}$ in $\mathcal{D}_{L^{\infty}, d}^{\left\{M_{p}\right\}}$.

In [16], the following assertion concerning the product of functions in $\mathcal{D}_{L^{\infty}, d}^{\left\{M_{p}\right\}}$ is proved:

Proposition 3.2 If $\varphi_{1}, \varphi_{2} \in \mathcal{D}_{L^{\infty}, d}^{\left\{M_{p}\right\}}$, then $\varphi_{1} \cdot \varphi_{2} \in \mathcal{D}_{L^{\infty}, d}^{\left\{M_{p}\right\}}$. Moreover, for every $\left(r_{p}\right) \in \mathfrak{R}$ such that $r_{1}>2$ the inequality holds:

$$
\left\|\varphi_{1} \cdot \varphi_{2}\right\|_{\left(r_{p}\right)} \leq\left\|\varphi_{1}\right\|_{\left(r_{p}\right) / 2} \cdot\left\|\varphi_{2}\right\|_{\left(r_{p}\right) / 2},
$$

where $\left(r_{p}\right) / 2$ is meant in the sense of (2.4).

Remark 3.1 The assertion of Proposition 3.2 is true for functions $\varphi_{1}, \varphi_{2}$ from the space $\mathcal{D}_{d}^{\left\{M_{p}\right\}}$ and semi-norms (3.5).

Remark 3.2 We may assume, if necessary, that the considered sequence $\left(r_{p}\right) \in \mathfrak{R}$ satisfies for any given constant $c>0$ the inequality $r_{p}>c$ for all $p \in \mathbb{N}$. In fact, we have $\|\varphi\|_{\left(r_{p}\right)}<\infty$ if and only if $\|\varphi\|_{\left(\widetilde{r}_{p}\right)}<\infty$ for all $\varphi \in \mathcal{D}_{d}^{\left\{M_{p}\right\}}$ with the sequence $\left(\widetilde{r}_{p}\right) \in \mathfrak{R}$ defined by $\widetilde{r}_{0}=1$ and $\widetilde{r}_{p}:=r_{p+p_{0}}$ for all $p \in \mathbb{N}$, where $p_{0} \in \mathbb{N}$ is an index such that $r_{p}>c$ for $p>p_{0}$.

\section{Roumieu ultradistributions}

Definition 4.1 We denote the strong dual of the space $\mathcal{D}_{d}^{\left\{M_{p}\right\}}$ by $\mathcal{D}_{d}^{\prime\left\{M_{p}\right\}}$ and call it the space of Roumieu ultradistributions. 
The strong dual of the space $\dot{\mathcal{B}}_{d}^{\left\{M_{p}\right\}}$, denoted by $\mathcal{D}_{L^{1}, d}^{\prime\left\{M_{p}\right\}}$, is called the space of Roumieu integrable ultradistributions.

Remark 4.1 The space $\mathcal{D}^{\left\{M_{p}\right\}}$ is dense in $\dot{\mathcal{B}}^{\left\{M_{p}\right\}}$ and the respective inclusion mapping is continuous. Consequently, the space $\mathcal{D}_{L^{1}}^{\left\{M_{p}\right\}}$ of Roumieu integrable ultradistributions is a subspace of the space $\mathcal{D}^{\prime\left\{M_{p}\right\}}$ of Roumieu ultradistributions.

Definition 4.2 By an $\mathfrak{R}$-approximate unit, we mean a sequence $\left(\Pi_{n}\right)$ of ultradifferentiable functions $\Pi_{n} \in \mathcal{D}_{d}^{\left\{M_{p}\right\}}$ converging to 1 in $\mathcal{E}_{d}^{\left\{M_{p}\right\}}$ such that the following property holds for every sequence $\left(r_{p}\right) \in \mathfrak{R}$ :

$$
\sup _{n \in \mathbb{N}}\left\|\Pi_{n}\right\|_{\left(r_{p}\right)}=\sup _{n \in \mathbb{N}_{k \in \mathbb{N}_{0}^{d}}}\left(R_{|k|} M_{k}\right)^{-1}\left\|D^{k} \Pi_{n}\right\|_{\infty}<\infty,
$$

where $\left(R_{p}\right)$ is the product sequence corresponding to $\left(r_{p}\right)$.

Definition 4.3 By a special $\mathfrak{R}$-approximate unit, we mean an $\mathfrak{R}$-approximate unit $\left(\Pi_{n}\right)$ such that for every compact set $K \subset \mathbb{R}^{d}$, there exists an index $n_{0} \in \mathbb{N}$ such that $\Pi_{n}(x)=1$ for all $n \geq n_{0}$ and $x \in K$.

We denote the class of all $\mathfrak{R}$-approximate units on $\mathbb{R}^{d}$ by $\mathbb{U}_{d}^{\left\{M_{p}\right\}}$ and the class of all special $\mathfrak{R}$-approximate units on $\mathbb{R}^{d}$ by $\overline{\mathbb{U}}_{d}^{\left\{M_{p}\right\}}$.

Remark 4.2 By the Denjoy-Carleman theorem, the defined above spaces of ultradifferentiable functions as well as the classes $\mathbb{U}_{d}^{\left\{M_{p}\right\}}$ and $\overline{\mathbb{U}}_{d}^{\left\{M_{p}\right\}}$ of approximate units contain sufficiently many members.

\section{Integrability of Roumieu ultradistributions}

We formulate below a characterization of integrable Roumieu ultradistributions, in the form of five equivalent conditions, which is an analog of the theorem of Dierolf and Voigt concerning integrable distributions (see [5]) and of the theorem of Pilipović concerning ultradistributions of Beurling type (see [18]). The proof of the theorem is given in [16].

Theorem 5.1 Let $V \in \mathcal{D}_{d}^{\prime\left\{M_{p}\right\}}$. The following conditions are equivalent:

(A) $V \in \mathcal{D}_{L^{1}, d}^{\prime\left\{M_{p}\right\}}$, i.e., $V$ is continuous on $\mathcal{D}_{d}^{\left\{M_{p}\right\}}$ in the topology induced by $\dot{\mathcal{B}}_{d}^{\left\{M_{p}\right\}}$, which means that there are a sequence $\left(r_{p}\right) \in \mathfrak{R}$ and a constant $C>0$ such that the inequality

$$
|\langle V, \varphi\rangle| \leq C\|\varphi\|_{\left(r_{p}\right)}
$$

holds for all $\varphi \in \mathcal{D}_{d}^{\left\{M_{p}\right\}}$; 
(B) there is a sequence $\left(r_{p}\right) \in \mathfrak{R}$ with the property that for every $\varepsilon>0$, there exists a regular compact set $K \subset \mathbb{R}^{d}$ such that the inequality

$$
|\langle V, \varphi\rangle| \leq \varepsilon\|\varphi\|_{\left(r_{p}\right)}
$$

holds for $\varphi \in \mathcal{D}_{d}^{\left\{M_{p}\right\}}$ with supp $\varphi \cap K=\emptyset$;

(C) for every $\left(\Pi_{n}\right) \in \mathbb{U}_{d}^{\left\{M_{p}\right\}}$ the sequence $\left(\left\langle V, \Pi_{n}\right\rangle\right)$ is Cauchy;

(D) for every $\left(\bar{\Pi}_{n}\right) \in \overline{\mathbb{U}}_{d}^{\left\{M_{p}\right\}}$ the sequence $\left(\left\langle V, \bar{\Pi}_{n}\right\rangle\right)$ is Cauchy;

(E) there are a sequence $\left(r_{p}\right) \in \mathfrak{R}$, a constant $C>0$ and a regular compact $K \subset \mathbb{R}^{d}$ such that inequality (5.1) holds for $\varphi \in \mathcal{D}_{d}^{\left\{M_{p}\right\}}$ with $\operatorname{supp} \varphi \cap K=\emptyset$.

Moreover, if $V$ satisfies any of the conditions (A)-(E), then

$$
\lim _{n \rightarrow \infty}\left\langle V, \Pi_{n}\right\rangle=\lim _{n \rightarrow \infty}\left\langle V, \bar{\Pi}_{n}\right\rangle=\langle V, 1\rangle
$$

for arbitrary $\left(\Pi_{n}\right) \in \mathbb{U}_{d}^{\left\{M_{p}\right\}}$ and $\left(\overline{\{\Pi}_{n}\right) \in \overline{\mathbb{U}}_{d}^{\left\{M_{p}\right\}}$.

\section{Convolution of Roumieu ultradistributions}

Pilipović and Prangoski made in [19] a deep study of the convolution of Roumieu ultradistributions. The study was based on the investigation of the $\epsilon$ tensor product of the respective spaces of test functions. Let us recall some results proved and observations made in [19].

The authors use the results on the $\varepsilon$ tensor product from [14] to prove that

$$
\dot{\mathcal{B}}_{d_{1}}^{\left\{M_{p}\right\}} \varepsilon \dot{\mathcal{B}}_{d_{2}}^{\left\{M_{p}\right\}} \cong \dot{\mathcal{B}}_{d_{1}}^{\left\{M_{p}\right\}} \widehat{\otimes}_{\varepsilon} \dot{\mathcal{B}}_{d_{2}}^{\left\{M_{p}\right\}}
$$

in the sense of an isomorphism. They consider, analogously to ideas applied in [17] to the convolution of measures, the following semi-norms in the space $\mathcal{D}_{L^{\infty}, d}^{\left\{M_{p}\right\}}$ :

$$
q_{g,\left(r_{p}\right)}(\varphi):=\sup _{k \in \mathbb{N}_{0}^{d}} \sup _{x \in \mathbb{R}^{d}} \frac{\left|g(x) D^{k} \varphi(x)\right|}{R_{|k|} M_{k}}, \quad \varphi \in \mathcal{D}_{L^{\infty}, d}^{\left\{M_{p}\right\}} .
$$

Denote by $\widetilde{\mathcal{D}}_{L^{\infty}, d}^{\left\{M_{p}\right\}}$ the 1.c.s. $\mathcal{D}_{L^{\infty}, d}^{\left\{M_{p}\right\}}$ equipped with the topology defined by the family $\left\{q_{g,\left(r_{p}\right)}: g \in \mathcal{C}_{0},\left(r_{p}\right) \in \mathfrak{R}\right\}$ of semi-norms and the strong dual of $\widetilde{\widetilde{\mathcal{D}}}_{L^{\infty}, d}^{\left\{M_{p}\right\}}$ by $\left(\widetilde{\widetilde{\mathcal{D}}}_{L^{\infty}, d}^{\left\{M_{p}\right\}}\right)_{b}^{\prime}$. Relations between the strong dual of $\widetilde{\widetilde{\mathcal{D}}}_{L^{\infty}, d}^{\left\{M_{p}\right\}}$ and the space $\mathcal{D}_{L^{1}, d}^{\prime\left\{M_{p}\right\}}$ of integrable Roumieu ultradistributions were studied in [19]. The results obtained in [19] were then improved in [21], where the equality

$$
\left(\widetilde{\widetilde{\mathcal{D}}}_{L^{\infty}, d}^{\left\{M_{p}\right\}}\right)_{b}^{\prime}=\mathcal{D}_{L^{1}, d}^{\prime\left\{M_{p}\right\}}
$$

was proved in the sense of 1.c.s. (see [21, Prop. 5.3 and Prop. 5.4]). 
The obtained results allowed the authors to give in [19] the following definitions of convolvability and the convolution of two Roumieu ultradistributions, analogous to the Schwartz definition of the convolution of distributions (see [22]):

Definition 6.1 Let $S, T \in \mathcal{D}_{d}^{\prime\left\{M_{p}\right\}}$. If the following condition is satisfied:

$$
V_{\varphi}:=(S \otimes T) \varphi^{\triangle} \in \mathcal{D}_{L^{1}, 2 d}^{\prime\left\{M_{p}\right\}} \quad \text { for all } \varphi \in \mathcal{D}_{d}^{\left\{M_{p}\right\}},
$$

where $\varphi^{\triangle}$ is the function of the class $\mathcal{E}_{2 d}^{\left\{M_{p}\right\}}$ defined by

$$
\varphi^{\triangleright}(x, y):=\varphi(x+y), \quad x, y \in \mathbb{R}^{d},
$$

we say that the Roumieu ultradistributions $S, T$ are convolvable in the sense of $(\mathrm{S})$. If $S, T$ are convolvable, then the convolution $S * T$ of $S$ and $T$ in $\mathcal{D}_{d}^{\prime\left\{M_{p}\right\}}$ is defined by

$$
\langle S * T, \varphi\rangle_{d}:=\left\langle V_{\varphi}, 1_{2 d}\right\rangle_{2 d}, \quad \varphi \in \mathcal{D}_{d}^{\left\{M_{p}\right\}}
$$

where $V_{\varphi}$ is meant, according to (6.1), as an element of the space $\widetilde{\widetilde{D}}_{L^{\infty}, 2 d}^{\prime\left\{M_{p}\right\}}$ and the constant function $1_{2 d}$ is meant as an element of the space $\widetilde{\widetilde{\mathcal{D}}}_{L^{\infty}, 2 d}^{\left\{M_{p}\right\}}$.

For each $a>0$ consider the subset $\triangle_{a}:=\left\{(x, y) \in \mathbb{R}^{2 d}:|x+y| \leq a\right\}$ of $\mathbb{R}^{2 d}$ and the following subspace of $\dot{\mathcal{B}}_{2 d}^{\left\{M_{p}\right\}}$ :

$$
\dot{\mathcal{B}}_{2 d}^{\left\{M_{p}\right\}}\left(\triangle_{a}\right):=\left\{\varphi \in \dot{\mathcal{B}}_{2 d}^{\left\{M_{p}\right\}}: \operatorname{supp} \varphi \subseteq \triangle_{a}\right\} .
$$

Denote by $\dot{\mathcal{B}}_{\triangle, 2 d}^{\left\{M_{p}\right\}}$ the inductive limit of the spaces defined in (6.4):

$$
\dot{\mathcal{B}}_{\triangle, 2 d}^{\left\{M_{p}\right\}}:=\lim _{a \rightarrow \infty} \dot{\mathcal{B}}_{2 d}^{\left\{M_{p}\right\}}\left(\triangle_{a}\right) .
$$

The following result on equivalence of convolvability conditions for Roumieu ultradistributions was proved in [19]:

Theorem 6.1 Let $S, T \in \mathcal{D}_{d}^{\prime\left\{M_{p}\right\}}$. The following conditions are equivalent to condition ( $\mathrm{S})$ of convolvability for $S$ and $T$ :

(c) $\quad S \otimes T \in \dot{\mathcal{B}}_{\triangle, 2 d}^{\left\{M_{p}\right\}}$;

$\left(c_{1}^{\prime}\right) \quad S(\check{T} * \varphi) \in \mathcal{D}_{L^{1}, d}^{\prime\left\{M_{p}\right\}}$ for all $\varphi \in \mathcal{D}_{d}^{\left\{M_{p}\right\}}$ and

$$
\mathcal{D}_{K, d}^{\left\{M_{p}\right\}} \times \dot{\mathcal{B}}_{d}^{\left\{M_{p}\right\}} \ni(\varphi, \chi) \mapsto\langle S(\check{T} * \varphi), \chi\rangle \in \mathbb{C}
$$

is a continuous bilinear mapping for every compact subset $K$ of $\mathbb{R}^{d}$; 
$\left(c_{2}^{\prime}\right) \quad(\check{S} * \varphi) T \in \mathcal{D}_{L^{1}, d}^{\prime\left\{M_{p}\right\}}$ for all $\varphi \in \mathcal{D}_{d}^{\left\{M_{p}\right\}}$ and

$$
\mathcal{D}_{K, d}^{\left\{M_{p}\right\}} \times \dot{\mathcal{B}}_{d}^{\left\{M_{p}\right\}} \ni(\varphi, \chi) \mapsto\langle(\check{S} * \varphi) T, \chi\rangle \in \mathbb{C}
$$

is a continuous bilinear mapping for every compact subset $K$ of $\mathbb{R}^{d}$;

$\left(c_{3}\right) \quad(\check{S} * \varphi)(T * \psi) \in L_{d}^{1}$ for all $\varphi, \psi \in \mathcal{D}_{d}^{\left\{M_{p}\right\}}$.

Dimovski et al. modified conditions $\left(c_{1}^{\prime}\right)$ and $\left(c_{2}^{\prime}\right)$ and obtained in [7] and [21] results (under the conditions (M.1), (M.2) and (M.3) imposed on $\left(M_{p}\right)$ ), which can be described in the form of the following theorem (see Theorem 8.2 in [7] and Remark 5.9. in [21]):

Theorem 6.2 Let $S, T \in \mathcal{D}_{d}^{\prime\left\{M_{p}\right\}}$. Each of the following two conditions is equivalent to condition $(\mathrm{S})$ of convolvability for $S$ and $T$ :

$\left(c_{1}\right) \quad S(\check{T} * \varphi) \in \mathcal{D}_{L^{1}, d}^{\prime\left\{M_{p}\right\}}$ for all $\varphi \in \mathcal{D}_{d}^{\left\{M_{p}\right\}}$;

$\left(c_{2}\right) \quad(\check{S} * \varphi) T \in \mathcal{D}_{L^{1}, d}^{\prime\left\{M_{p}\right\}}$ for all $\varphi \in \mathcal{D}_{d}^{\left\{M_{p}\right\}}$.

Moreover, if any of the conditions $(\mathrm{S}),\left(c_{1}\right),\left(c_{2}\right)$ holds, then

$$
\langle S * T, \varphi\rangle_{d}=\left\langle{ }_{d} S(\check{T} * \varphi), 1_{d}\right\rangle_{d}=\left\langle(\check{S} * \varphi) T, 1_{d}\right\rangle_{d}
$$

for all $\varphi \in \mathcal{D}_{d}^{\left\{M_{p}\right\}}$.

In the next section, we are going to formulate several sequential conditions of convolvability of Roumieu ultradistributions, which are equivalent to conditions $(\mathrm{S}),\left(c_{1}\right)$ and $\left(c_{2}\right)$.

\section{Sequential convolutions of Roumieu ultradistributions}

The notion of $\mathfrak{R}$-approximate unit makes it possible to consider several sequential definitions of the convolution of Roumieu ultradistributions based on corresponding sequential conditions of convolvability. The conditions require that respective numerical sequences, corresponding to a given pair of Roumieu ultradistributions via certain approximate units, are Cauchy sequence (Cauchy s. in short) for all approximate units from a given class. The first definition of this kind was given for the convolution of distributions by Vladimirov in [23] and its equivalent versions were discussed in [5] and [9]. Their counterparts for ultradistributions of Beurling type were discussed in [10] (see also [1]).

We will prove in Theorem 7.1 that all the sequential definitions are equivalent to the definition of the general convolution of Roumieu ultradistributions in the sense of Pilipović and Prangoski [19]. Our proof of Theorem 7.1 will be based on the integrability result stated in the previous section. 
Definition 7.1 Let $S, T \in \mathcal{D}_{d}^{\prime\left\{M_{p}\right\}}$. We say that $S, T$ are convolvable in the sense of $(\mathrm{V}),(\Pi),\left(\Pi_{1}\right),\left(\Pi_{2}\right)$, respectively, if the corresponding condition below holds for every $\varphi \in \mathcal{D}_{d}^{\left\{M_{p}\right\}}$ :

(V) $\left(\left\langle S \otimes T, \Pi_{n} \varphi^{\Delta}\right\rangle_{2 d}\right)$ is a Cauchy s. for all $\left(\Pi_{n}\right) \in \mathbb{U}_{2 d}^{\left\{M_{p}\right\}}$;

(ח) $\left(\left\langle\left(\Pi_{n}^{1} S\right) \otimes\left(\Pi_{n}^{2} T\right), \varphi^{\Delta}\right\rangle_{2 d}\right)$ is a Cauchy s. for all $\left(\Pi_{n}^{1}\right),\left(\Pi_{n}^{2}\right) \in \mathbb{U}_{d}^{\left\{M_{p}\right\}}$;

$\left(\Pi_{1}\right) \quad\left(\left\langle\left(\Pi_{n} S\right) \otimes T, \varphi^{\Delta}\right\rangle_{2 d}\right) \quad$ is a Cauchy s. for all $\left(\Pi_{n}\right) \in \mathbb{U}_{d}^{\left\{M_{p}\right\}}$;

$\left(\Pi_{2}\right) \quad\left(\left\langle S \otimes\left(\Pi_{n} T\right), \varphi^{\Delta}\right\rangle_{2 d}\right)$ is a Cauchy s. for all $\left(\Pi_{n}\right) \in \mathbb{U}_{d}^{\left\{M_{p}\right\}}$,

respectively.

Definition 7.2 If $S, T \in \mathcal{D}_{d}^{\prime\left\{M_{p}\right\}}$ are convolvable in the sense of $(\mathrm{V}),(\Pi),\left(\Pi_{1}\right),\left(\Pi_{2}\right)$, respectively, then the convolution of $S$ and $T$ in $\mathcal{D}_{d}^{\prime\left\{M_{p}\right\}}$ in the respective sense is defined by the corresponding formula below:

$$
\begin{array}{lll}
\langle S * T, \varphi\rangle_{d}:=\lim _{n \rightarrow \infty}\left\langle S \otimes T, \Pi_{n} \varphi^{\Delta}\right\rangle_{2 d}, & \varphi \in \mathcal{D}_{d}^{\left\{M_{p}\right\}}, & \left(\Pi_{n}\right) \in \mathbb{U}_{2 d}^{\left\{M_{p}\right\}} ; \\
\langle S * T, \varphi\rangle_{d}:=\lim _{n \rightarrow \infty}\left\langle\left(\Pi_{n}^{1} S\right) \otimes\left(\Pi_{n}^{2} T\right), \varphi^{\Delta}\right\rangle_{2 d}, & \varphi \in \mathcal{D}_{d}^{\left\{M_{p}\right\}}, \quad\left(\Pi_{n}^{1}\right),\left(\Pi_{n}^{2}\right) \in \mathbb{U}_{d}^{\left\{M_{p}\right\}} ; \\
\left\langle S_{*}^{\Pi_{1}} T, \varphi\right\rangle_{d}:=\lim _{n \rightarrow \infty}\left\langle\left(\Pi_{n} S\right) \otimes T, \varphi^{\Delta}\right\rangle_{2 d}, & \varphi \in \mathcal{D}_{d}^{\left\{M_{p}\right\}}, \quad\left(\Pi_{n}\right) \in \mathbb{U}_{d}^{\left\{M_{p}\right\}} ; \\
\left\langle S_{*}^{\Pi_{2}} T, \varphi\right\rangle_{d}:=\lim _{n \rightarrow \infty}\left\langle S \otimes\left(\Pi_{n} T\right), \varphi^{\Delta}\right\rangle_{2 d}, & \varphi \in \mathcal{D}_{d}^{\left\{M_{p}\right\}}, \quad\left(\Pi_{n}\right) \in \mathbb{U}_{d}^{\left\{M_{p}\right\}} ;
\end{array}
$$

respectively.

Remark 7.1 Condition (S) of convolvability in Definition 6.1 guarantees, by the considerations of Pilipović and Prangoski from Sect. 4 in [19], that the convolution $S * T$ in the sense of (6.3) exists in $\mathcal{D}_{d}^{\prime\left\{M_{p}\right\}}$. It follows from Theorem 7.1, formulated below, that the convolvability conditions $(\mathrm{V}),(\Pi),\left(\Pi_{1}\right)$ and $\left(\Pi_{2}\right)$ guarantee that the corresponding sequential convolutions, defined in Definition 6, exist in $\mathcal{D}_{d}^{\prime\left\{M_{p}\right\}}$.

In addition to the sequential conditions of convolvability in $\mathcal{D}_{d}^{\prime\left\{M_{p}\right\}}$, given in Definition 7.1 , one may consider also the conditions $(\bar{V}),(\bar{\Pi}),\left(\bar{\Pi}_{1}\right),\left(\bar{\Pi}_{2}\right)$ being the modifications of the above ones, which consist in replacing the classes $\mathbb{U}_{2 d}^{\left\{M_{p}\right\}}$ and $\mathbb{U}_{d}^{\left\{M_{p}\right\}}$ of $\mathfrak{R}$-approximate units by the classes $\overline{\mathbb{U}}_{2 d}^{\left\{M_{p}\right\}}$ and $\overline{\mathbb{U}}_{d}^{\left\{M_{p}\right\}}$ of special approximate units, respectively. The modified conditions lead to additional sequential definitions of the convolution in $\mathcal{D}_{d}^{\prime\left\{M_{p}\right\}}$ which are counterparts of the known sequential definitions of the convolution of distributions (see [5, 9, 23]). It follows from Theorem 5.1 that they are equivalent to all the conditions listed in Definition 7.1.

Remark 7.2 The convolution of Roumieu ultradistributions in $\mathcal{D}_{d}^{\prime\left\{M_{p}\right\}}$ investigated in [19] and [7] and its sequential versions discussed in this paper is a general notion. 
It embraces various particular cases, e.g., expressed in terms of supports of given Roumieu ultradistributions.

Remark 7.3 Each of the sequential definitions of the convolution of $S$ and $T$, given in Definition 7.2 under the corresponding conditions, do not depend on the choice of an approximate unit from the class $\mathbb{U}^{\left\{M_{p}\right\}}\left(\mathbb{U}_{2 d}^{\left\{M_{p}\right\}}\right.$ or $\mathbb{U}_{d}^{\left\{M_{p}\right\}}$, respectively), because

$$
\left(\Pi_{n}^{j}\right) \in \mathbb{U}^{\left\{M_{p}\right\}} \quad \text { for } \quad j \in\{1,2\} \Longrightarrow\left(\Pi_{n}\right) \in \mathbb{U}^{\left\{M_{p}\right\}},
$$

where $\Pi_{2 n-1}:=\Pi_{n}^{1}$ and $\Pi_{2 n}:=\Pi_{n}^{2}$ for $n \in \mathbb{N}$.

Theorem 7.1 Let $S, T \in \mathcal{D}_{d}^{\prime\left\{M_{p}\right\}}$. All the conditions formulated in Definition 7.1 are equivalent to the condition (S) of convolvability of ultradistributions. If one of these conditions is satisfied, then all corresponding convolutions of Roumieu ultradistributions $S$ and $T$ exist in $\mathcal{D}_{d}^{\prime\left\{M_{p}\right\}}$ and the following equalities hold true:

$$
S * T=S * T=S^{\Pi} \Pi_{1}^{\Pi_{2}} T=S * T=S * T .
$$

Proof We will prove the equivalence of convolvability conditions given in Definitions 7.1 and 6.1 and in Theorem 6.2 according to the following scheme of implications:

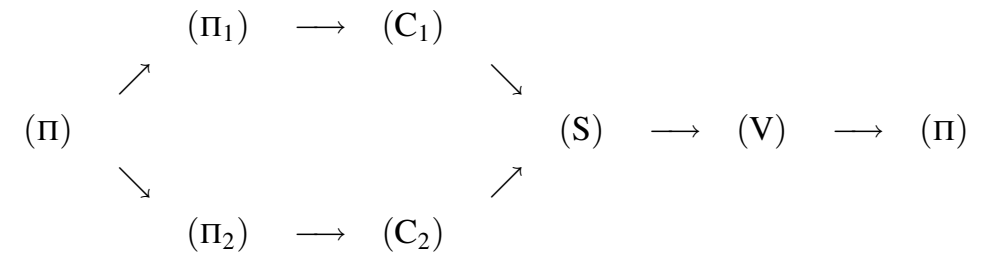

Assume first condition (П) for a fixed $\varphi \in \mathcal{D}_{d}^{\left\{M_{p}\right\}}$. Due to Remark 7.3, there exists an $\alpha \in \mathbb{C}$ such that

$$
\lim _{n \rightarrow \infty}\left\langle\left(\Pi_{n}^{1} S\right) \otimes\left(\Pi_{n}^{2} T\right), \varphi^{\Delta}\right\rangle_{2 d}=\alpha
$$

for all $\left(\Pi_{n}^{1}\right),\left(\Pi_{n}^{2}\right) \in \mathbb{U}_{d}^{\left\{M_{p}\right\}}$. Hence, the double limit

$$
\lim _{i, j \rightarrow \infty}\left\langle\left(\Pi_{i}^{1} S\right) \otimes\left(\Pi_{j}^{2} T\right), \varphi^{\Delta}\right\rangle_{2 d}=\alpha
$$

also exists for all $\left(\Pi_{i}^{1}\right),\left(\Pi_{j}^{2}\right) \in \mathbb{U}_{d}^{\left\{M_{p}\right\}}$. Indeed, if (7.2) were not true, then there would exist an $\varepsilon>0$ and increasing sequences $\left(i_{n}\right)$ and $\left(j_{n}\right)$ of positive integers such that 


$$
\left|\left\langle\left(\Pi_{i_{n}}^{1} S\right) \otimes\left(\Pi_{j_{n}}^{2} T\right), \varphi^{\Delta}\right\rangle-\alpha\right|>\varepsilon
$$

for all $n \in \mathbb{N}$, but this would contradicts condition (ח), because $\left(\Pi_{i_{n}}^{1}\right),\left(\Pi_{j_{n}}^{2}\right) \in \mathbb{U}_{d}^{\left\{M_{p}\right\}}$. On the other hand, we have

$$
\lim _{m \rightarrow \infty}\left\langle\left(\Pi_{n}^{1} S\right) \otimes\left(\Pi_{m}^{2} T\right), \varphi^{\Delta}\right\rangle_{2 d}=\left\langle\left(\Pi_{n}^{1} S\right) \otimes T, \varphi^{\Delta}\right\rangle_{2 d}
$$

for every fixed $n \in \mathbb{N}$, because $\Pi_{m}^{2} T \rightarrow T$ in $\mathcal{D}_{d}^{\prime\left\{M_{p}\right\}}$ as $m \rightarrow \infty$ and

$$
\operatorname{supp}\left[\left(\left(\Pi_{n}^{1} S\right) \otimes\left(\Pi_{m}^{2} T\right)\right) \cdot \varphi^{\Delta}\right] \subset \operatorname{supp}\left[\left(\left(\Pi_{n}^{1} S\right) \otimes T\right) \cdot \varphi^{\Delta}\right]
$$

for all $n, m \in \mathbb{N}$, while the support of $\left[\left(\Pi_{n}^{1} S\right) \otimes T\right] \cdot \varphi^{\triangle}$ is compact for every $n \in \mathbb{N}$. Clearly, (7.2) and (7.3) imply

$$
\lim _{n \rightarrow \infty}\left\langle\left(\Pi_{n}^{1} S\right) \otimes T, \varphi^{\Delta}\right\rangle_{2 d}=\alpha .
$$

Consequently, condition $\left(\Pi_{1}\right)$ is satisfied. Moreover, equalities (7.1) and (7.4) yield the equality

$$
\langle S * T, \varphi\rangle_{d}=\left\langle S^{\Pi_{1}} T, \varphi\right\rangle_{d}
$$

for all $\varphi \in \mathcal{D}_{d}^{\left\{M_{p}\right\}}$. Thus, we have proved that (ח) implies $\left(\Pi_{1}\right)$ and equality (7.5) holds under condition (П).

Assume now condition $\left(\Pi_{1}\right)$ and fix arbitrarily $\varphi \in \mathcal{D}_{d}^{\left\{M_{p}\right\}}$ and $\left(\Pi_{n}\right) \in \mathbb{U}_{d}^{\left\{M_{p}\right\}}$. The equality

$$
\left\langle\left(\Pi_{n} S\right) \otimes T, \varphi^{\Delta}\right\rangle_{2 d}=\left\langle\left(\Pi_{n} S\right) * T, \varphi\right\rangle_{d}=\left\langle S(\check{T} * \varphi), \Pi_{n}\right\rangle_{d}, \quad n \in \mathbb{N},
$$

and condition $\left(\Pi_{1}\right)$ imply that the sequence $\left(\left\langle S(\check{T} * \varphi), \Pi_{n}\right\rangle_{d}\right)$ is Cauchy, so $S(\check{T} *$ $\varphi)$ is integrable for all $\varphi \in \mathcal{D}_{d}^{\left\{M_{p}\right\}}$, by Theorem 5.1. The same equality yields

$$
\lim _{n \rightarrow \infty}\left\langle\left(\Pi_{n} S\right) \otimes T, \varphi^{\Delta}\right\rangle_{2 d}=\left\langle S(\check{T} * \varphi), 1_{d}\right\rangle_{d}
$$

for all $\left(\Pi_{n}\right) \in \mathbb{U}_{d}^{\left\{M_{p}\right\}}$ and $\varphi \in \mathcal{D}_{d}^{\left\{M_{p}\right\}}$, i.e.,

$$
\langle S * T, \varphi\rangle_{d}=\left\langle S(\check{T} * \varphi), 1_{d}\right\rangle_{d}
$$

for all $\varphi \in \mathcal{D}_{d}^{\left\{M_{p}\right\}}$. Consequently, we have shown the implication $\left(\Pi_{1}\right) \Rightarrow\left(c_{1}\right)$ and that (7.6) is true under the assumption of condition $\left(\Pi_{1}\right)$.

From the above, by symmetry, we conclude the implications $(\Pi) \Rightarrow\left(\Pi_{2}\right)$ and $\left(\Pi_{2}\right) \Rightarrow\left(c_{2}\right)$ as well as the equalities

$$
\langle S * T, \varphi\rangle_{d}=\left\langle S^{\Pi} * T, \varphi\right\rangle_{d}, \quad \varphi \in \mathcal{D}_{d}^{\left\{M_{p}\right\}}
$$

and 


$$
\left\langle S^{\Pi_{2}} T, \varphi\right\rangle_{d}=\left\langle(\check{S} * \varphi) T, 1_{d}\right\rangle_{d}, \quad \varphi \in \mathcal{D}_{d}^{\left\{M_{p}\right\}},
$$

which are true under conditions $(\Pi)$ and $\left(\Pi_{2}\right)$, respectively.

By Theorem 6.2, we have $\left(c_{1}\right) \Leftrightarrow\left(c_{2}\right) \Leftrightarrow(\mathrm{S})$ and the equalities in (6.5) hold true, if any of the three conditions is satisfied. Hence, by (7.6) and (7.8), we have

$$
\langle S * T, \varphi\rangle_{d}=\left\langle S * T, 1_{d}\right\rangle, \quad \varphi \in \mathcal{D}_{d}^{\left\{M_{p}\right\}},
$$

under the assumption of condition $\left(c_{j}\right)$ for $j \in\{1,2\}$.

Notice that also conditions (S) and (V) are equivalent and each of these conditions implies

$$
\langle S * T, \varphi\rangle_{d}=\langle S * T, \varphi\rangle_{d}
$$

for $\varphi \in \mathcal{D}_{d}^{\left\{M_{p}\right\}}$. Equality (7.10) is an immediate consequence of Theorem 5.1 applied for $V:=(S \otimes T) \varphi^{\triangle}$ with $\varphi \in \mathcal{D}_{d}^{\left\{M_{p}\right\}}$ and for $\left(\Pi_{n}\right) \in \mathbb{U}_{2 d}^{\left\{M_{p}\right\}}$.

Finally, assume condition (V) and fix arbitrarily $\left(\Pi_{n}^{1}\right),\left(\Pi_{n}^{2}\right) \in \mathbb{U}_{d}^{\left\{M_{p}\right\}}$. Then $\left(\Pi_{n}\right) \in \mathbb{U}_{2 d}^{\left\{M_{p}\right\}}$ and $\Pi_{n} \varphi^{\Delta} \in \mathcal{D}_{2 d}^{\left\{M_{p}\right\}}$ for $n \in \mathbb{N}$ and $\varphi \in \mathcal{D}_{d}^{\left\{M_{p}\right\}}$, where $\Pi_{n}:=\Pi_{n}^{1} \otimes \Pi_{n}^{2}$ for $n \in \mathbb{N}$. Applying a simple property of the tensor product in the spaces $\mathcal{D}_{d}^{\left\{M_{p}\right\}}$ and $\mathcal{D}_{d}^{\prime\left\{M_{p}\right\}}$ as well as topological isomorphisms described in Theorems 2.1 and 2.3 in [13], we get the equality:

$$
\left\langle\left(\Pi_{n}^{1} S\right) \otimes\left(\Pi_{n}^{2} T\right), \varphi^{\Delta}\right\rangle_{2 d}=\left\langle S \otimes T, \Pi_{n} \varphi^{\Delta}\right\rangle_{2 d}
$$

for all $\varphi \in \mathcal{D}_{d}^{\left\{M_{p}\right\}}$ and $n \in \mathbb{N}$. Due to this equality, (V) implies condition ( $\Pi$ ) and thus

$$
\langle S * T, \varphi\rangle_{d}=\langle S * T, \varphi\rangle_{d}
$$

for $\varphi \in \mathcal{D}_{d}^{\left\{M_{p}\right\}}$. Consequently, the last implication $(\mathrm{V}) \Rightarrow(\Pi)$ in the scheme and equality (7.11), under the assumption of condition (V), are proved.

This completes the proof that all conditions in Definition 7.1 are equivalent and, moreover, equalities (7.5)-(7.11) show that all the convolutions defined in Definition 7.2 are equal to the convolution $S * T$ defined in (6.3), whenever any of the considered convolvability conditions is satisfied.

Remark 7.4 In addition, one may consider the sequential conditions $(\overline{\mathrm{V}}),(\bar{\Pi}),\left(\bar{\Pi}_{1}\right)$, $\left(\bar{\Pi}_{2}\right)$ of convolvability of $S, T \in \mathcal{D}_{d}^{\prime\left\{M_{p}\right\}}$ and the corresponding convolutions $S * T$, $S * T, S * T, S_{*}^{\overline{\Pi_{2}}} T$ of $S$ and $T$, replacing the classes $\mathbb{U}_{2 d}^{\left\{M_{p}\right\}}$ and $\mathbb{U}_{d}^{\left\{M_{p}\right\}}$ of $\mathfrak{R}$ approximate units by the classes $\overline{\mathbb{U}}_{2 d}^{\left\{M_{p}\right\}}$ and $\overline{\mathbb{U}}_{d}^{\left\{M_{p}\right\}}$ of special $\mathfrak{R}$-approximate units.

The equivalence of conditions $(\mathrm{S}),(\overline{\mathrm{V}}),(\bar{\Pi}),\left(\bar{\Pi}_{1}\right)$ and $\left(\bar{\Pi}_{2}\right)$ of convolvability follows in the same manner as the equivalence of conditions $(\mathrm{S}),(\mathrm{V}),(\Pi),\left(\Pi_{1}\right)$ and $\left(\Pi_{2}\right)$ proved in Theorem 7.1 above. The equality of the corresponding convolutions is an easy consequence of this result. 
Remark 7.5 It is easy to see that the convolution of Roumieu ultradistributions is commutative, i.e., $S * T=T * S$ for $S, T \in \mathcal{D}_{d}^{\prime\left\{M_{p}\right\}}$.

\section{Ultradifferential property of the convolution}

Let us consider an ultradifferential operator $P(D)$ defined by Komatsu in [12] as follows:

Definition 8.1 An operator of the form

$$
P(D)=\sum_{k \in \mathbb{N}_{0}^{d}} c_{k} D^{k}, \quad c_{k} \in \mathbb{C}
$$

is called an ultradifferential operator of class $\left\{M_{p}\right\}$ if for every $L>0$ there is a constant $C_{L}$ such that

$$
\left|c_{k}\right| \leq C_{L} \frac{L^{k}}{M_{k}}, \quad k \in \mathbb{N}_{0}^{d}
$$

Clearly, the condition in Definition 8.1 can be equivalently expressed as follows

$$
\forall_{L>0} \quad \sup _{k}\left(L^{-k} M_{k}\left|c_{k}\right|\right)<\infty .
$$

Then according to Lemma 2.1, part (II), there is a sequence $\left(u_{p}\right) \in \mathfrak{R}$ such that $\sup _{k}\left(U_{|k|} M_{k}\left|c_{k}\right|\right)<\infty$, where $U_{k}=\prod_{p \leq k} u_{p}$. In other words, an ultradifferential operator of the form (8.1) is of class $\left\{M_{p}\right\}$ if there are $C>0$ and $\left(u_{p}\right) \in \mathfrak{R}$ such that

$$
\left|c_{k}\right| \leq \frac{C}{U_{|k|} M_{k}}, \quad k \in \mathbb{N}_{0}^{d}
$$

Due to Komatsu [12], if $\left(M_{p}\right)$ satisfies condition (M.2), then $P(D)$ defines the respective continuous mappings $\mathcal{D}_{d}^{\left\{M_{p}\right\}} \rightarrow \mathcal{D}_{d}^{\left\{M_{p}\right\}}$ and $\mathcal{D}_{d}^{\prime\left\{M_{p}\right\}} \rightarrow \mathcal{D}_{d}^{\prime\left\{M_{p}\right\}}$. Moreover, the series $P(D) S=\sum_{k \in \mathbb{N}_{0}^{d}} c_{k} D^{k} S$ converges absolutely in $\mathcal{D}_{d}^{\prime\left\{M_{p}\right\}}$ for every $S \in \mathcal{D}_{d}^{\prime\left\{M_{p}\right\}}$.

In Theorem 8.1 below we prove an important and non-trivial property of the convolution of Roumieu ultradistributions. In the proof, we will need the following very useful result from [19]:

Lemma 8.1 For every sequence $\left(s_{p}\right) \in \mathfrak{R}$, there exists a sequence $\left(r_{p}\right) \in \mathfrak{R}$ such that $r_{p} \leq s_{p}$ for $p \in \mathbb{N}$ and

$$
R_{p+q} \leq 2^{p+q} R_{p} R_{q} \quad \text { for all } p, q \in \mathbb{N}_{0} .
$$


Theorem 8.1 Let $S, T \in \mathcal{D}_{d}^{\prime\left\{M_{p}\right\}}$ be convolvable and let $P(D)$ be an ultradifferential operator of class $\left\{M_{p}\right\}$. Then $P(D) S$ and $T$ as well as $S$ and $P(D) T$ are convolvable and, moreover,

$$
P(D)(S * T)=(P(D) S) * T=S *(P(D) T) .
$$

Proof Assume that $S, T \in \mathcal{D}_{d}^{\prime\left\{M_{p}\right\}}$ are convolvable. By the definitions of $P(D)$ and $S * T$ and by Theorem 5.1, we have

$$
\begin{aligned}
\langle P(D)(S * T), \varphi\rangle & =\langle S * T, P(-D) \varphi\rangle=\left\langle(S \otimes T)\left(P\left(-D_{x}\right) \varphi\right)^{\Delta}, 1_{2 d}\right\rangle \\
& =\lim _{n \rightarrow \infty}\left\langle S \otimes T, \Pi_{n}\left[P\left(-D_{x}\right) \varphi^{\Delta}\right]\right\rangle
\end{aligned}
$$

for all $\left(\Pi_{n}\right) \in \overline{\mathbb{U}}_{2 d}^{\left\{M_{p}\right\}}$ and $\varphi \in \mathcal{D}_{d}^{\left\{M_{p}\right\}}$.

It suffices to prove that the ultradistributions $P(D) S$ and $T$ are convolvable in $\mathcal{D}_{d}^{\prime\left\{M_{p}\right\}}$ and that the first equality of (8.4) holds. The remaining part of the assertion follows then directly from Remark 7.5. To prove the convolvability of $P(D) S$ and $T$, we have to show that the sequence $\left(\left\langle P(D) S \otimes T, \Pi_{n} \varphi^{\Delta}\right\rangle\right)_{n \in \mathbb{N}}$ is convergent.

We have

$$
\begin{aligned}
\left\langle P(D) S \otimes T, \Pi_{n} \varphi^{\Delta}\right\rangle & =\sum_{\alpha \in \mathbb{N}_{0}^{d}} c_{\alpha}\left\langle D_{x}^{\alpha} S \otimes T, \Pi_{n} \varphi^{\Delta}\right\rangle=\sum_{\alpha \in \mathbb{N}_{0}^{d}} c_{\alpha}\left\langle S \otimes T,-D_{x}^{\alpha}\left(\Pi_{n} \varphi^{\Delta}\right)\right\rangle \\
& =\left\langle S \otimes T, P\left(-D_{x}\right)\left(\Pi_{n} \varphi^{\Delta}\right)\right\rangle
\end{aligned}
$$

for all $n \in \mathbb{N}$, by (8.1) and the absolute convergence of the respective series.

Comparing the last terms in (8.5) and (8.6), we see that the declared assertion will be proved if we show the equalities

$$
P\left(-D_{x}\right)\left(\Pi_{n} \varphi^{\Delta}\right)=\Pi_{n} P\left(-D_{x}\right) \varphi^{\Delta}+v_{n}
$$

on $\mathbb{R}^{2 d}$ for all $n \in \mathbb{N}$, where $v_{n}$ are certain functions in $\mathcal{D}_{d}^{\left\{M_{p}\right\}}$ such that

$$
\lim _{n \rightarrow \infty}\left\langle S \otimes T, v_{n}\right\rangle=0 .
$$

Applying (8.1) and Leibniz' rule and then changing the order of summation, we get, for all $n \in \mathbb{N}$, the equalities

$$
\begin{aligned}
P\left(-D_{x}\right)\left(\Pi_{n} \varphi^{\Delta}\right) & =\sum_{\alpha \in \mathbb{N}_{0}^{d}}(-1)^{|\alpha|} c_{\alpha} \sum_{i \leq \alpha}\left(\begin{array}{c}
\alpha \\
i
\end{array}\right)\left(D_{x}^{i} \Pi_{n}\right)\left(D^{\alpha-i} \varphi\right)^{\Delta} \\
& =\sum_{i \in \mathbb{N}_{0}^{d}} D_{x}^{i} \Pi_{n} \sum_{\beta \in \mathbb{N}_{0}^{d}}(-1)^{|\beta+i|}\left(\begin{array}{c}
\beta+i \\
i
\end{array}\right) c_{\beta+i}\left(D^{\beta} \varphi\right)^{\Delta} \\
& =\Pi_{n} P\left(-D_{x}\right) \varphi^{\Delta}+v_{n}
\end{aligned}
$$


on $\mathbb{R}^{2 d}$ with the functions $v_{n}$ defined for $x, y \in \mathbb{R}^{d}$ by

$$
v_{n}(x, y):=\sum_{i \in \mathcal{N}} D_{x}^{i} \Pi_{n}(x, y) \sum_{\beta \in \mathbb{N}_{0}^{d}}(-1)^{|\beta+i|}\left(\begin{array}{c}
\beta+i \\
i
\end{array}\right) c_{\beta+i} D_{x}^{\beta} \varphi(x+y),
$$

where $\mathcal{N}:=\mathbb{N}_{0}^{d} \backslash\{(0, \ldots, 0)\}$. This means that equations (8.7) hold for $v_{n}$ defined in (8.9) and for all $n \in \mathbb{N}$. Clearly, $\left(v_{n}\right)$ depends on the initial sequence $\left(\Pi_{n}\right)$.

It suffices to show (8.8). Choose $\theta \in \mathcal{D}_{d}^{\left\{M_{p}\right\}}$ such that $\theta(x)=1$ for $x \in \operatorname{supp} \varphi$. By (8.9), we have

$$
\left\langle S \otimes T, v_{n}\right\rangle=\left\langle(S \otimes T) \theta^{\triangle}, v_{n}\right\rangle, \quad n \in \mathbb{N} .
$$

The sequence $\left(\left\langle(S \otimes T) \theta^{\Delta}, \bar{\Pi}_{n}\right\rangle\right)_{n \in \mathbb{N}}$ is convergent for every $\left(\bar{\Pi}_{n}\right) \in \overline{\mathbb{U}}_{2 d}^{\left\{M_{p}\right\}}$, by the assumption that $S$ and $T$ are convolvable in $\mathcal{D}_{d}^{\prime\left\{M_{p}\right\}}$. To prove (8.8) it is enough to show that also $\left(\bar{\Pi}_{n}+v_{n}\right) \in \overline{\mathbb{U}}_{2 d}^{\left\{M_{p}\right\}}$. Since $\left(\Pi_{n}\right),\left(\bar{\Pi}_{n}\right) \in \overline{\mathbb{U}}_{2 d}^{\left\{M_{p}\right\}}$, for each compact set $K$ in $\mathbb{R}^{2 d}$ there exists an $n_{0} \in \mathbb{N}$ such that $D^{i} \Pi_{n}(x, y)=0$ and $\bar{\Pi}_{n}(x, y)=1$ for $(x, y) \in K, i \in \mathcal{N}$ and $n>n_{0}$. Consequently, in view of (8.9),

$$
\bar{\Pi}_{n}(x, y)+v_{n}(x, y)=1 \quad \text { for }(x, y) \in K, n>n_{0} .
$$

Therefore it remains to prove, for every $\left(t_{p}\right) \in \mathfrak{R}$, that

$$
\sup _{n \in \mathbb{N}}\left\|v_{n}\right\|_{\left(t_{p}\right)}<\infty
$$

since $\left(\bar{\Pi}_{n}\right) \in \overline{\mathbb{U}}_{2 d}^{\left\{M_{p}\right\}}$ and (8.10) implies

$$
\sup _{n \in \mathbb{N}}\left\|\bar{\Pi}_{n}+v_{n}\right\|_{\left(t_{p}\right)}<\infty .
$$

Fix an arbitrary $\left(t_{p}\right) \in \mathfrak{R}$. The coefficients of the ultradifferential operator $P(D)$ satisfy (8.2) for some $\left(u_{p}\right) \in \mathfrak{R}$. Putting $s_{k}:=\min \left\{t_{k}, u_{k}\right\}$ for $k \in \mathbb{N}$, we have $\left(s_{p}\right) \in \mathfrak{R}$. By Lemma 8.1, there exists a sequence $\left(r_{p}\right) \in \mathfrak{R}$ such that $r_{k} \leq s_{k}$ and inequality (8.3) holds. In addition we assume, according to Remark 3.2, that

$$
r_{p}>16 H^{2} \quad \text { for } \quad p \in \mathbb{N}
$$

where $H>\frac{1}{4}$ is a constant from condition (M.2).

Let $\alpha \in \mathbb{N}_{0}^{d}$ be arbitrarily fixed. For fixed $n \in \mathbb{N}$ and $\left(r_{p}\right) \in \mathfrak{R}$ chosen above, according to the representation (8.9), we have 


$$
\begin{gathered}
\frac{\left\|D_{x}^{\alpha} v_{n}\right\|_{\infty}}{R_{|\alpha|} M_{\alpha}} \leq \sum_{i \in \mathcal{N}} \sum_{j \leq \alpha}\left(\begin{array}{c}
\alpha \\
j
\end{array}\right) \frac{\left\|D_{x}^{\alpha+i-j} \Pi_{n}\right\|_{\infty}}{R_{|\alpha+i-j|} M_{\alpha+i-j}} \sum_{\beta \in \mathbb{N}_{0}^{d}}\left(\begin{array}{c}
\beta+i \\
i
\end{array}\right)\left|c_{\beta+i}\right| \\
\cdot \frac{\left\|D_{x}^{\beta+j} \varphi^{\Delta}\right\|_{\infty}}{R_{|\beta+j|} M_{\beta+j}} \cdot \frac{R_{|\alpha+i-j|} R_{|\beta+j|}}{R_{|\alpha|}} \cdot \frac{M_{\alpha+i-j} M_{\beta+j}}{M_{\alpha}}
\end{gathered}
$$

Applying properties (2.3) and (8.3) (twice) of the sequence $\left(r_{p}\right)$ and property (2.1) and condition (M.2) (twice) of the sequence $\left(M_{p}\right)$, we get

$$
\frac{R_{|\alpha+i-j|} R_{|\beta+j|}}{R_{|\alpha|} R_{|\beta|} R_{|i|}} \leq \frac{2^{|\beta+j|} R_{|\alpha+i|}}{R_{|\alpha|} R_{|i|}} \leq 2^{|\alpha+i|} 2^{|\beta+j|}
$$

and

$$
\frac{M_{\alpha+i-j} M_{\beta+j}}{M_{\alpha} M_{\beta} M_{i}} \leq \frac{A H^{|\beta|+|j|} M_{\alpha+i}}{M_{\alpha} M_{i}} \leq A^{2} H^{|\alpha+i|} H^{|\beta+j|} .
$$

Moreover, we have

$$
\left|c_{\beta+i}\right| \leq \frac{C}{U_{|\beta|} U_{|i|} M_{\beta} M_{i}} \quad \text { and } \quad \frac{R_{|\beta|} R_{|i|}}{U_{|\beta|} U_{|i|}} \leq 1,
$$

by (8.2), properties (2.1) of $\left(M_{p}\right)$ and (2.3) of $\left(u_{p}\right) \in \mathfrak{R}$ and because $r_{k} \leq u_{k}$ for $k \in \mathbb{N}$. The inequalities in (8.13), (8.14) and (8.15) hold for arbitrary $\alpha, \beta, i, j \in \mathbb{N}_{0}^{d}$ such that $j \leq \alpha$ and will be used later together with the following known estimate:

$$
\left(\begin{array}{c}
\beta+i \\
i
\end{array}\right) \leq 2^{|\beta+i|},
$$

for a certain $B>0$ and all $\beta, i \in \mathbb{N}_{0}^{d}$.

It follows from (8.12), due to (8.13)-(8.16), that

$$
\begin{aligned}
& \frac{\left\|D_{x}^{\alpha} v_{n}\right\|_{\infty}}{R_{|\alpha|} M_{\alpha}} \leq A^{2} C \sum_{i \in \mathcal{N}} \sum_{j \leq \alpha}\left(\begin{array}{c}
\alpha \\
j
\end{array}\right)(2 H)^{|\alpha+i|} \frac{\left\|D_{x}^{\alpha+i-j} \Pi_{n}\right\|_{\infty}}{R_{|\alpha+i-j|} M_{\alpha+i-j}} \\
& \cdot \sum_{\beta \in \mathbb{N}_{0}^{d}} 2^{|\beta+i|}(2 H)^{|\beta+j|} \frac{\left\|D_{x}^{\beta+j} \varphi^{\Delta}\right\|_{\infty}}{R_{|\beta+j|} M_{\beta+j}} .
\end{aligned}
$$

According to assumption (8.11), consider the sequences $\left(\bar{r}_{p}\right)$ and $\left(\overline{\bar{r}}_{p}\right)$ of the class $\mathfrak{R}$ defined by $\bar{r}_{p}:=r_{p} / 8 H$ and $\bar{r}_{p}:=r_{p} / 16 H^{2}$, respectively, for $p \in \mathbb{N}$. Clearly,

$$
2^{2|\alpha+i-j|}(2 H)^{|\alpha+i-j|} \frac{\left\|D_{x}^{\alpha+i-j} \Pi_{n}\right\|_{\infty}}{R_{|\alpha+i-j|} M_{\alpha+i-j}} \leq\left\|\Pi_{n}\right\|_{\left(\bar{r}_{p}\right)}
$$

and 


$$
2^{2|\beta+j|}(2 H)^{2|\beta+j|} \frac{\left\|D_{x}^{\beta+j} \varphi^{\Delta}\right\|_{\infty}}{R_{|\beta+j|} M_{\beta+j}} \leq\|\varphi\|_{\left(\overline{\bar{r}}_{p}\right)}
$$

for all $\alpha, \beta, i, j \in \mathbb{N}_{0}^{d}, j \leq \alpha$. We deduce from (8.17) and the above estimates that

$$
\frac{\left\|D_{x}^{\alpha} v_{n}\right\|_{\infty}}{R_{|\alpha|} M_{\alpha}} \leq \frac{A^{2} C}{2^{\alpha}}\|\Pi\|_{\left(\bar{r}_{p}\right)}\|\varphi\|_{\left(\overline{\bar{r}}_{p}\right)} \cdot \sum_{i \in \mathcal{N}}\left(\frac{1}{2}\right)^{i} \sum_{\beta \in \mathbb{N}_{0}^{d}}\left(\frac{1}{4 H}\right)^{\beta}<\infty .
$$

for arbitrary $\alpha \in \mathbb{N}_{0}^{d}$ and $n \in \mathbb{N}$. Hence

$$
\sup _{n \in \mathbb{N}}\left\|v_{n}\right\|_{\left(r_{p}\right)}=\sup _{n \in \mathbb{N}} \sup _{\alpha \in \mathbb{N}_{0}^{d}} \frac{\left\|D_{x}^{\alpha} v_{n}\right\|_{\infty}}{R_{|\alpha|} M_{\alpha}}<\infty
$$

and, since $r_{p} \leq t_{p}$ for $p \in \mathbb{N}$,

$$
\sup _{n \in \mathbb{N}}\left\|v_{n}\right\|_{\left(t_{p}\right)} \leq \sup _{n \in \mathbb{N}}\left\|v_{n}\right\|_{\left(r_{p}\right)}<\infty
$$

i.e., (8.10) is proved, as required. The assertion of Theorem 8.1 is proved.

Theorem 8.1 has also been shown in the quasianalytic case in the article [21]. The proof there is given via a completely different method (cf. [21, Cor. 5.10]).

Acknowledgements The author would like to thank sincerely the anonymous referee for clear-sighted remarks and helpful comments. This work was partly supported by the Center for Innovation and Transfer of Natural Sciences and Engineering Knowledge of University of Rzeszów.

Open Access This article is licensed under a Creative Commons Attribution 4.0 International License, which permits use, sharing, adaptation, distribution and reproduction in any medium or format, as long as you give appropriate credit to the original author(s) and the source, provide a link to the Creative Commons licence, and indicate if changes were made. The images or other third party material in this article are included in the article's Creative Commons licence, unless indicated otherwise in a credit line to the material. If material is not included in the article's Creative Commons licence and your intended use is not permitted by statutory regulation or exceeds the permitted use, you will need to obtain permission directly from the copyright holder. To view a copy of this licence, visit http:// creativecommons.org/licenses/by/4.0/.

\section{References}

1. Carmichael, R.D., Kamiński, A., Pilipović, S.: Boundary Values and Convolution in Ultradistribution Spaces. World Scientific, London (2007)

2. Debrouwere, A., Vindas, J.: On weighted inductive limits of spaces of ultradifferentiable functions and their duals. Math. Nachr. 292(3), 573-602 (2019)

3. Debrouwere, A., Vindas, J.: A projective description of generalized Gelfand-Shilov spaces of Roumieu type. Analysis, Probabilility, Applications, and Computation. Trends in Mathematics, pp. 407-417. Birkhäuser, Cham (2019) 
4. Debrouwere, A., Prangoski, B., Vindas, J.: On the projective description of spaces of ultradifferentiable functions of Roumieu type. Preprint (2019). arXiv:1912.11938 [math.FA]

5. Dierolf, P., Voigt, J.: Convolution and $\mathcal{S}^{\prime}$-convolution of distributions. Collect. Math. 29, 185-196 (1978)

6. Dimovski, P., Pilipović, S., Vindas, J.: New distribution spaces associated to translation-invariant Banach spaces. Monatsh. Math. 177, 495-515 (2015)

7. Dimovski, P., Pilipović, S., Prangoski, B., Vindas, J.: Convolution of ultradistributions and ultradistribution spaces associated to translation-invariant Banach spaces. Kyoto J. Math. 56(2), 401-440 (2016)

8. Dimovski, P., Prangoski, B., Vindas, J.: On a class of translation-invariant spaces of quasianalytic ultradistributions. Novi Sad J. Math. 45(1), 143-175 (2015)

9. Kamiński, A.: Convolution, product and Fourier transform of distributions. Stud. Math. 74, 83-86 (1982)

10. Kamiński, A., Kovačević, D., Pilipović, S.: The equivalence of various definitions of the convolution of ultradistributions. Trudy Mat. Inst. Steklov 203, 307-322 (1994)

11. Kamiński, A., Perišić, D., Pilipović, S.: Existence theorems for convolution of ultradistributions. Dissertationes Math. 340, 93-114 (1995)

12. Komatsu, H.: Ultradistributions, I: structure theorems and a characterization. J. Fac. Sci. Univ. Tokyo Sect. IA Math. 20, 25-105 (1973)

13. Komatsu, H.: Ultradistributions, II: the kernel theorem and ultradistribution with support submanifold. J. Fac. Sci. Univ. Tokyo Sect. IA Math. 24, 607-628 (1977)

14. Komatsu, H.: Ultradistributions, III: vector valued ultradistributions and the theory of kernels. J. Fac. Sci. Univ. Tokyo, Sect. IA Math. 29, 653-717 (1982)

15. Mincheva-Kaminska, S.: Convolution of distributions in sequential approach. Filomat 28(8), 1543-1557 (2014)

16. Mincheva-Kaminska, S.: Sequential conditions of integrability of Roumieu ultradistributions. Preprint (2019). arXiv:1910.14173v2 [math.FA]

17. Ortner, N., Wagner, P.: Distribution-Valued Analytic Functions-Theory and Applications. MaxPlanck-Institute, Leipzig (2008)

18. Pilipović, S.: On the convolution in the space of Beurling ultradistributions. Comment. Math. Univ. St. Paul 40, 15-27 (1991)

19. Pilipović, S., Prangoski, B.: On the convolution of Roumieu ultradistributions through the $\varepsilon$ tensor product. Monatsh. Math. 173, 83-105 (2014)

20. Pilipović, S., Prangoski, B.: Anti-Wick and Weyl quantization on ultradistribution spaces. J. Math. Pure Appl. 103, 472-503 (2015)

21. Pilipović, S., Prangoski, B., Vindas, J.: On quasianalytic classes of Gelfand-Shilov type. Parametrix and convolution. J. Math. Pures Appl. 116, 174-210 (2018)

22. Schwartz, L.: Définitions integrale de la convolution de deux distributions. in: Séminaire Schwartz, Année 1953-54. Expose ${ }^{\circ}$ 22, Secr. math. Fac. Sci., Paris (1954)

23. Vladimirov, V.S.: Equations of Mathematical Physics, Nauka, Moscow 1967 (in Russian), English edn. Marcel Dekker, New York (1971)

24. Vučković, D., Vindas, J.: Eigenfunction expansions of ultradifferentiable functions and ultradistributions in $\mathbb{R}^{n}$. J. Pseudo Differ. Oper. Appl. 7, 519-531 (2016) 